\title{
Acute Retinal Necrosis Caused by Co-infection of Herpes Simplex Virus and Varicella-Zoster Virus: Case Report
}

\section{Kasra Cheraqpour}

Tehran University of Medical Sciences

Aliasghar Ahmadraji ( $\sim$ hamidraji2000@yahoo.com )

Tehran University of Medical Sciences https://orcid.org/0000-0003-1371-0813

\section{Ali Rashidinia}

TUMS: Tehran University of Medical Sciences

Maziyar Irannejad

TUMS: Tehran University of Medical Sciences

\section{Brief report}

Keywords: Acute retinal necrosis, ARN, Herpes Simplex Virus, Varicella-Zoster Virus

Posted Date: October 7th, 2020

DOI: https://doi.org/10.21203/rs.3.rs-86138/v1

License: (c) (i) This work is licensed under a Creative Commons Attribution 4.0 International License. Read Full License 


\section{Abstract}

Background: Acute retinal necrosis is considered as a rare infectious uveitis. This condition is usually caused by Varicella-zoster virus or Herpes simplex virus. At the time being, acute retinal necrosis caused by co-infection of multiple viruses is sorely rare report. Herein, we report an immunocompetent patient who developed ARN with co-infection of Herpes Simplex Virus (type I and II) and Varicella-Zoster Virus (VZV).

Findings: An adult immunocompetent man presented with complain of decreased vision of the right eye from 12 days before. Examination of the right eye revealed severe conjunctival injection, fine diffuse keratic precipitates, 3+ anterior chamber and vitreous cells, slightly elevated intraocular pressure (26), blurred optic disk with hemorrhagic patches, and occlusive vasculitis plus confluent necrotizing patches on the peripheral retina compatible with diagnosis of acute retinal necrosis. The patient has been admitted and underwent for sampling from intra-ocular fluids which showed presence of HSV I \& II and VZV on real-Time PCR of vitreous specimens confirmed by rechecking. We used of intravenous acyclovir followed by oral acyclovir and oral prednisolone to control the disease which was successful.

Conclusions: Although rare, we speculate multi viral infection may be considered in the physiopathology of acute retinal necrosis.

\section{Introduction}

Acute retinal necrosis (ARN) is a rare infectious uveitis usually caused by some members of the herpes virus family [1]. In 2007, Muthiah MN et al. reported an incidence rate of one case per 1.6 to 2 million population of UK per year [2]. ARN may occur in both immunocompetent and immunesuppressed individuals. There is no sexual predilection in patients and most of the cases occur between the fifth and seventh decades of life [3]. 2/3 of patients experience unilateral involvement whereas in $1 / 3$ of cases the disease may spread to the fellow-eye within 1-6 weeks after the first eye involvement. Bilateral occurrence of ARN is more common in neonates and immunosuppressive conditions [3]. The involved eyes may be complicated with retinal detachment (RD), macular ischemia, and optic atrophy[4]. Although diagnosis of ARN is clinically, polymerase chain reaction (PCR) test as a highly specific method is routinely performed on specimens of intra-ocular fluids in suspected cases to recognize viral DNA [5].

To date, ARN caused by multiple viruses is an extremely rare finding. Herein, we describe an immunocompetent patient who developed ARN with co-infection of Herpes Simplex Virus (type I and II) and Varicella-Zoster Virus (VZV).

\section{Case Presentation}

A 54-year-old man presented to the emergency department of Farabi Eye Hospital with complaint of decreased vision of the right eye from 12 days before. His past medical history was positive for multiple sclerosis (MS). It should be mentioned the patient has been used Natalizumab to treating MS. At the 
presentation, his best-corrected visual acuity (BCVA) of the left eye was 20/20 and slit-lamp examination and funduscopy revealed no pathologic findings on the left eye (shown in Fig. 1). The right eye had a BCVA of 20/200, severe conjunctival injection, fine diffuse keratic precipitates (KPs), $3+$ anterior chamber (AC) and vitreous cells, slightly elevated intraocular pressure (26), blurred optic disk with hemorrhagic patches, and occlusive vasculitis plus confluent necrotizing patches on the peripheral retina (shown in Fig. 2). According to the clinical findings ARN was the most possible scenario. The patient has been admitted and scheduled for immediate AC and vitreous tap which revealed presence of HSV I \& II and VZV on real-Time PCR of vitreous samples. Target DNA was isolated and genotyped by Real-Time PCR with Taqman ${ }^{\mathrm{TM}}\left(\mathrm{AB|} \mid{ }^{\circledR}, \mathrm{USA}\right)$ and Hybridization probe (Roche ${ }^{\circledR}$, Germany) in a private laboratory. Intravenous acyclovir (10 mg/kg every 8 hours for 1 week) was used for the induction phase of the treatment. Response to the treatment was significant and inflammatory signs began to resolve. We used oral acyclovir adjunct to oral prednisolone on a tapering strategy for 3 months as the maintenance phase. It's noticeable we performed $360^{\circ}$ prophylactic laser photocoagulation to prevent probably subsequent RRD. However our attempt was not successful; the patient was complicated with refractory RRD which underwent for pars plana vitrectomy, silicone oil injection, and endolaser photocoagulation for multiple times. Despite all of used procedures the right eye did not gain a BCVA better than hand motions.

\section{Discussion}

Acute retinal necrosis (ARN) is a sight-threatening uveitis which commonly caused by Varicella-zoster virus (VZV), Herpes Simplex virus type 1 and 2 (HSV I and HSV II), Cytomegalovirus (CMV), and EpsteinBarr virus (EBV) [5]. Recently Hüseyin Baran Özdemir et al. reported a case of acute retinal necrosis presenting with central retinal vascular occlusion caused by human adenovirus [5]. Both immunocompetent and immunosuppressed individuals may develop ARN. Thus, properties of causative agent may be more determining than status of host's immune system [6]. Recent herpes virus infection, genetic factors, and immunosuppression may prone the patients to ARN [1]. In addition to corticosteroids, monoclonal antibodies such as Natalizumab using in the treatment of MS can be considered as a predisposing factor for developing ARN as occurred in our case [1].

Patients may complain ocular pain worsening with eye movements, red eye, decreased vision, and floaters [6]. Diagnosis of ARN is clinically; common clinical features include remarkable inflammatory reaction of both anterior chamber and vitreous, at least one foci of retinal necrosis in the peripheral retina with circumferential spread, rapid progression in the lack of appropriate treatment, and an arterial occlusive vasculopathy [5]. A well-defined, smooth, and geographic border separates necrotic retina from healthy parts. Also, scant areas of retinal hemorrhages may be present [6]. Despite clinical-based diagnosis, PCR technique on intraocular fluids is routinely used in practice to identify the causing virus [7].

The most commonly used antiviral agents in the treatment of ARN are intravenous acyclovir or oral valacyclovir[5]. Oral famciclovir, valganciclovir and acyclovir are the other useful drugs. It has been discussed fellow-eye involvement is frequent without antiviral therapy [5]. Although intravenous 
administration of high-dose acyclovir is recommended as the choice option of treatment, reports of usefulness of oral antiviral therapy are available. At the time being no studies on direct comparison between oral and intravenous routs of antiviral treatment has been conducted [7]. It is recommended the patient should be maintained on oral agents for 3-6 months to reduce the risk of fellow-eye involvement. However no clear strategy has been advised on duration and dosage of prophylaxis[7]. The role of corticosteroids in the treatment is controversial. Reducing the inflammation is accounted as their advantages whereas worsening the necrotizing retinopathy is a potential concern [3]. It is noticeable although acyclovir is effective in regression of the necrosis and also reducing the chance of second eye involvement, it does not have preventive role in developing of retinal detachment [6]. On this issue, it seems the role of performing prophylactic laser photocoagulation is controversial. In one side some studies reported laser photocoagulation posterior to the necrotic areas can decrease rate of retinal detachment about $50 \%$ and in the other side Sara Risseeuw et al. refused this result in condition of corrected disease severity and recommended future investigations to clarify the effects of prophylactic vitrectomy $[6,8]$.

Visual outcome is mainly poor due to undesirable sequels such as retinal detachment and ischemic accidents of the optic nerve or macula[7]. The chance of retinal detachment increases especially in cases with $>25 \%$ retinal necrosis [7].

To the best of our knowledge this is one of the extremely limited reports of the ARN with multiple viral infections. Previously, Shida Chen et al. reported a 52-year-old man with diagnosis of acute retinal necrosis caused by multiple viruses [9]. They initiated the treatment with high-dose oral valacyclovir which was successful. However their patient complicated with macular detachment at the 4th month of follow- up and underwent to sclera buckling and pars plana vitrectomy with a final vision of counting fingers to hand motions[9]. On the issue of viral DNA detection, they used two primer pairs on samples from diagnostic vitrectomy; the first one could detect the presence of HSV-1, HSV-2, EBV, or CMV and the second one could identify VZV, human herpes virus 6 (HHV-6), or HHV-7. Both primer pairs amplified showing infection by at least two different viruses. On the one hand, EBV and CMV are not common for developing ARN and on the other hand HHV-6 and HHV-7 are not discussed in pathogenesis of ARN. So the authors of mentioned case report concluded the most probably situation is co-infection of HSV and VZV. The other similar report was performed by Tomoko Nakamura et al. in 2015 [10]. They report identification of VZV and CMV DNA in the vitreous, tears, saliva, and skin of an old healthy woman. However they concluded according to clinical characteristics and response to acyclovir, CMV is not responsible for causing ARN in this woman. Unlike these two reports, results of PCR in our case showed exact simultaneous infection of three viruses (HSV-1, HSV-2, and VZV) which can be considered as the unique aspect of our report. To exclude the possibility of the lab error or contamination, we asked the laboratory to perform PCR on the specimens for the second time. The results were the same. Although this report was interesting and rare, it beseems multi viral infection did not affect the response to treatment in our case. Considering a separate role for multi viral infection in occurrence of refractory RRD is difficult since retinal detachment is a common complication of acute retinal necrosis. So, we speculate this finding has research values more than clinical. 


\section{Conclusion}

In conclusion, although rare, multi viral infection may be considered in the physiopathology of acute retinal necrosis.

\section{Abbreviations}

ARN: acute retinal necrosis; RD: retinal detachment; PCR: polymerase chain reaction; HSV: herpes simplex virus; VZV: Varicella-Zoster Virus; MS: multiple sclerosis; BCVA: best-corrected visual acuity; KP: keratic precipitate; $\mathrm{AC}$ : anterior chamber; RRD: rhegmatogenous retinal detachment; $\mathrm{CMV}$ : cytomegalovirus; $\mathrm{EBV}$ : epstein-barr virus; $\mathrm{HHV}$ : human herpes virus

\section{Declarations}

\section{Availability of data and materials}

The data is available from the corresponding author on reasonable request.

\section{Statement of Ethics}

N/A.

\section{Consent for publication}

Consent was taken from the individual for publication of personal data.

\section{Conflict of Interest Statement}

The authors declare no potential conflicts of interest.

\section{Funding Sources}

N/A.

\section{Author Contributions}

$A A$ visited the patient, performed treatment and collected the patient information. $K C$ wrote the manuscript. AA, AR, and MI performed critical revisions on manuscript. All authors read and approved final manuscript.

\section{ORCID iDs}

Kasra Cheraqpour https://orcid.org/0000-0002-1273-9166

\section{References}


1. Sood AB, Kumar G, Robinson J (2016) Bilateral acute retinal necrosis in a patient with multiple sclerosis on natalizumab. Journal of ophthalmic inflammation and infection 6 (1):1-4

2. Muthiah MN, Michaelides M, Child C, Mitchell SM (2007) Acute retinal necrosis: a national population-based study to assess the incidence, methods of diagnosis, treatment strategies and outcomes in the UK. British Journal of Ophthalmology 91 (11):1452-1455

3. Cruz GP, Fonseca C, Oliveira J, da Cunha JS (2019) Acute retinal necrosis by herpes simplex virus type 1: an unusual presentation of a primary infection. BMJ Case Reports CP 12 (12)

4. Chan EW, Sun V, Eldeeb M, Kapusta MA (2018) EPSTEIN-BARR VIRUS ACUTE RETINAL NECROSIS IN AN IMMUNOCOMPETENT HOST. Retinal Cases \& Brief Reports

5. Özdemir HB, Özdal PÇ (2020) Human Adenovirus: An Unusual Causative Agent for Acute Retinal Necrosis Presented with Central Retinal Vascular Occlusion. Ocular immunology and inflammation 28 (5):749-753

6. Walters G, James TE (2001) Viral causes of the acute retinal necrosis syndrome. Current opinion in ophthalmology 12 (3):191-195

7. Kobayashi T, Sekar P, Meier J, Streit J (2019) Acute retinal necrosis in a patient with remote severe herpes simplex encephalitis. BMJ Case Reports CP 12 (5):e229137

8. Risseeuw S, de Boer JH, Ninette H, van Leeuwen R (2019) Risk of rhegmatogenous retinal detachment in acute retinal necrosis with and without prophylactic intervention. American Journal of Ophthalmology 206:140-148

9. Chen S, Malik KJ, Shen D, Chan C-C (2014) Acute Retinal Necrosis with Multiple Viral Infections: A Case Report. International Journal of Ophthalmic Pathology 3 (5)

10. Nakamura T, Daikoku T, Shiraki K, Hayashi A (2015) Detection of cytomegalovirus in an immunocompetent adult presenting with acute retinal necrosis due to varicella-zoster virus: a case report. Clinical Ophthalmology (Auckland, NZ) 9:853

\section{Figures}




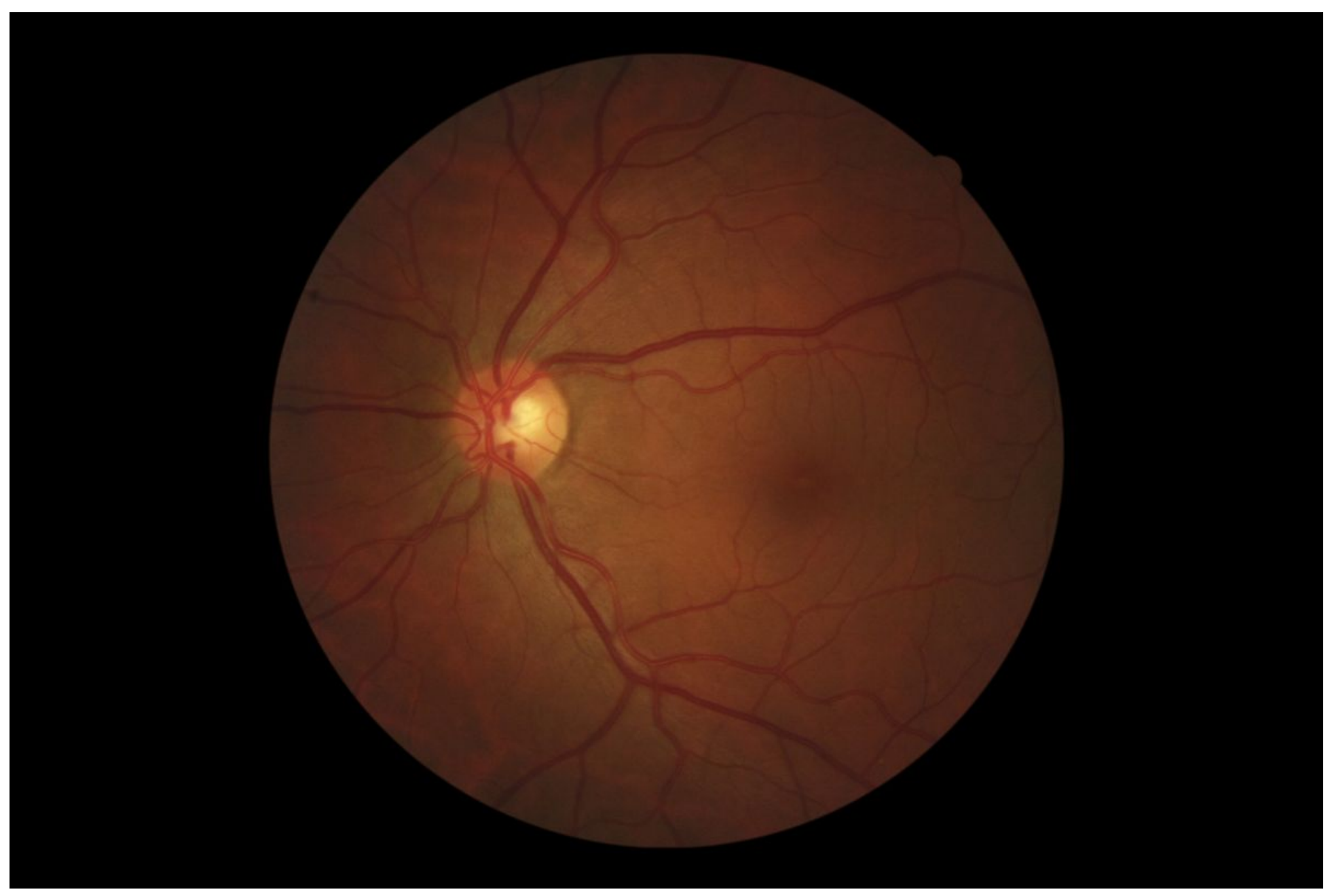

Figure 1

Normal fundus photograph of the left eye. 


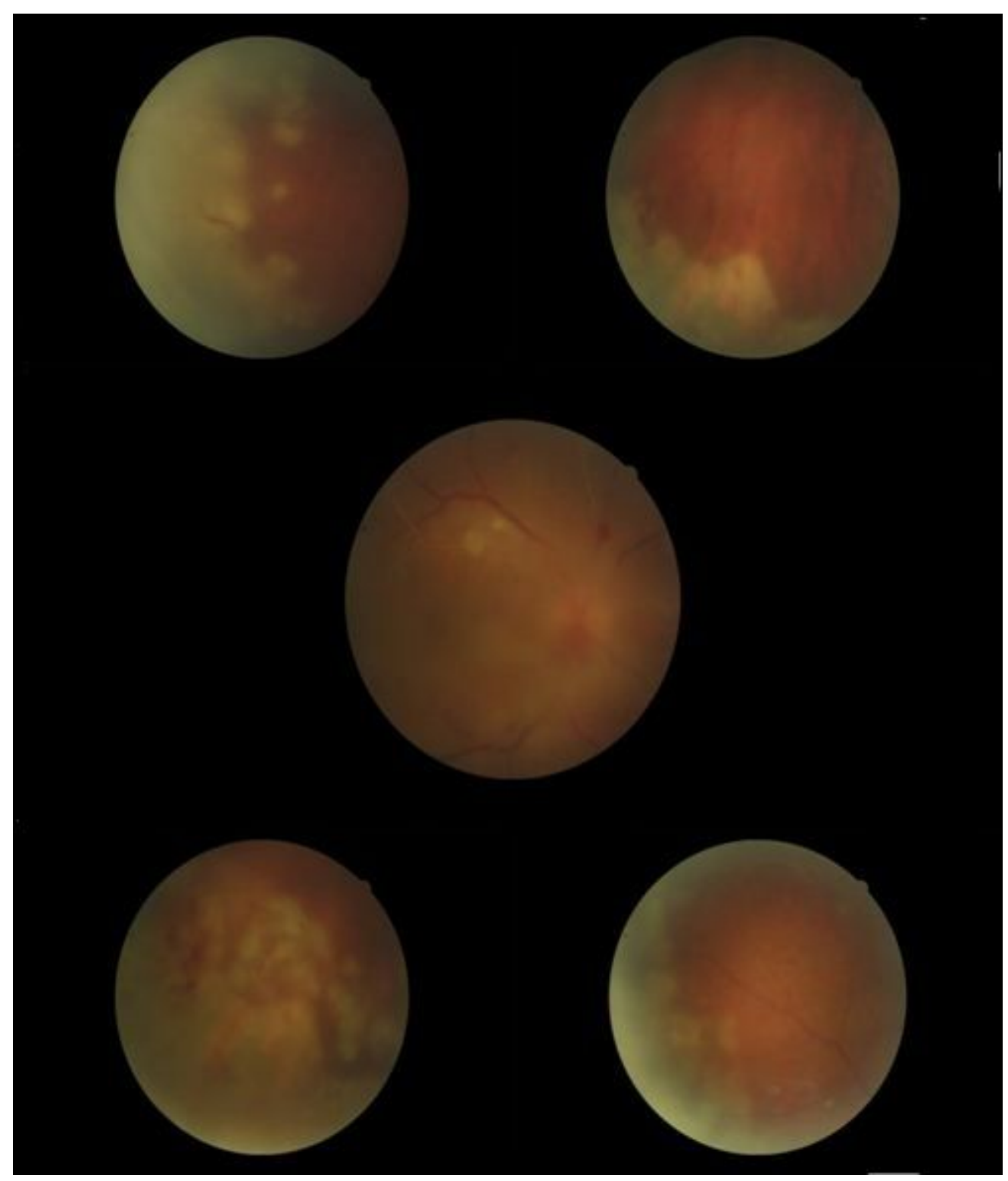

\section{Figure 2}

Fundus photograph of the left eye showing hazy media due to vitritis, blurred optic disk with hemorrhagic patches, occlusive vasculitis, confluent necrotizing patches on the peripheral retina, and laser spots. 